\title{
CONTRIBUCION AL CONOCIMIENTO DE LAS CONDICIONES HIDROGRAFICAS DE LOS FIORDOS DE LA REGION MAGALLANICA - CHILE
}

\author{
LISANDRO CHUECAS M. \\ Departamento de Biologfa Marina y Oceanograffa \\ Universidad de Concepción, Concepción, Chile \\ $y$ \\ RAMON AHUMADA B. \\ Departamento de Biologra y Tecnologra del Mar, \\ Pontificia Universidade Católica de Chile, Talcahuano, Chile
}

\section{SYNOPSIS}

The spring hydrographic conditions of Magellan fjords region were described (i.e., Seno Almirantazgo, Bahia Inutil, Seno Otway and Golfo Xaultegua). The large differences in the water body observed among the fiords were related to the distance between the fjords to the shore and the influence of the coastal waters. Furthemore a seasonal variations analysis with spring and fall data has been carried out. Tho result shows that the most important parameter in the seasonal fluctuations of the water body was temperature.

Introduccion

La Costa Austral de Ćhile se caracteríza por poseer una extensa zona de canales y fiordos que se inicia en los $42^{\circ}$ Lat. Sur y se extiende hasta el Cabo de Hornos. El conocimiento hidrográfico de esta región es relativamente pobre. Los primeros antecedentes se refieren a la zona Norte y fueron obtenidos por la Expedición LUND (Brattström) \& Dahl, 1951), Expedición Chiloé I (Chuecas, 1962) y Expedición Mar Chile I (Brandhorst, 1971). El afo 1970, la Expedición HUDSON-70, realizỏ un estudio sistemático de toda la región (Pickard 1971 y 1973) que aporta importantes antecedentes del área,

Posteriormente, Expedición HERO 72-4 repitió las estaciones hidrográficas realizadas el afio 1970 por la Expedición HUDSON con el propósito de comparar los resultados y estudiar las fluctuaciones estacionales de los cuerpos de aguas de los fiordos más australes.

En este trabajo se presenta los resultados de la Expedición HERO $72-4$.

\section{Material y Metodos}

Los estudios realizados en la Etapa I de la Expedición HERO $72-4$ (10 al 24 de Septiembre de 1972) comprendió 60 estaciones con un recorrido de 1.177 millas náuticas correspondientes a los fiordos ubicados entre Punta Arenas y Cabo Pilar. E1 trabajo se realizó a bordo del R/V "Hero" de la National Science Foundation y se obtuvo muestras en Seno Almirantazgo, Bahía Inútil, Seno Otway y Golfo Xaultegua, incluyendo estaciones en el Estrecho de Magallanes, Canal Gajardo y Caleta Elina (Fig. 1).

Las muestras de agua de mar para análisis de salinidad y oxígeno fueron obtenidas con botellas de inversión Nansen a diferentes profundidades y las temperaturas medidas con termómetros de inversión protegidos Kahlsico y Richter \& Wiese. La salinidad fue determinada en un salinómetro de inducción Auto-Lab, Mod MK III, con $0.003 \%$ 。 de precisión.

La valoración de oxígeno disuelto se hizo en muestras duplicadas por el método de Winkler, según Carritt \& Carpenter (1966).

\section{Resultados}

Para describir la distribución de los parámetros se utilizó diagramas de correlación T-S (Fig. 2) y $\mathrm{T}-\mathrm{O}_{2}$ (Fig. 3).

$\mathrm{El}$ análisis de correlación T-S superficial muestra amplios rangos de fluctación en Almirantazgo, Otway y Xaultegua. En cambio. Inútil presenta un rango de fluctuación pequefio.

Bn $20 \mathrm{~m}$ de profundidad, Almirantazgo presenta un aumento de la salinídad, comportamiento que lo asemeja a la distribución de Inútil: En cambio, Otway y Xaultegua presentan una capa más dihida con valores parecidos.

Em 50 m Otway, Inútil y Almirantazgo alcanzan la capa homotérmica e isohalina. No obstante, los valores absolutos de temperatura presentan diferencias significativas.

En el nivel de $100 \mathrm{~m}$ la temperatura y salinidad se estabilizan y la diferencia de los valores absolutos muestra un cambio en las características de las aguas de cada fiordo en particular. De acuerdo a esto, se puede apreciar una clara influencia de las aguas del Pacífico en Xaultegua. La salinidad en este nivel es de ca. $33.00 \%$ y la temperatura es de $c a .8 .5^{\circ} \mathrm{C}$; valores semejantes han sido registrados en las aguas fuera de los fiordos (Silva, 1977) Otway, Almirantazgo e Inútil presentan salinidades del orden de $30.50 \%$, donde el rango máximo de variación de salinidad entre ellos, es de $c a$. $0.20 \%$ o. Dependiendo la identidad de los cuerpos de agua de la temperatura. Las aguas de más de $100 \mathrm{~m}$ de profundidad mantienen la tendencia hidrográfica presentada para los fiordos estudiados.

En el análisis de correlación $\mathrm{T}-\mathrm{O}_{2}$ se usó las mismas profundidades que el diagrama T-S. El análisis no muestra diferencias significativas entre los diferentes fiordos y se puede hablar de una buena oxigenación en toda la columna de agua. La identidad de los cuerpos de agua en estos diagramas de correlación está dada por las diferencias de temperatura entre ellos en los diferentes niveles de profundidad,

Comparación de los resultados obtenidos por el HERO $72-4$ y el HUDSON 70.

$\mathrm{Tal}$ como se mencionó en la introducción, la Expedicion HERO $72-4$ repitió, para el área sur de los fiordos, las estaciones hidrográficas realizadas por la Expedición HUDSON 70. Sobre esa base y los períodos estacionales antípodas (i. e., Primavera-Otoño) en que se realiź los cruceros, se compara el comportamiento hidrográfico estacional del área.

La correlación TS para la capa superficial (Fig. 4) indica que la temperatura es el factor diferencial más importante en el comportamiento hidrográfico estacional. En otoffo la temperatura máxima fue de $9.86^{\circ} \mathrm{C}$ en Otway y la mínima de $8.0^{\circ} \mathrm{C}$, en C. Gajardo. En primavera en cambio, la máxima temperatura alcanza sólo a $5.4^{\circ} \mathrm{C}$ en Almirantazgo y la mínima de $4.8^{\circ} \mathrm{C}$ en Otway y C. Gajardo. La variación de salinidad presenta una mayor dilución en otorio, lo que permitiría suponer que el efecto de dilución corresponde a las aguas de deshielo. Esta apreciación se reforzania por la constancia de la salinidad en Inútil, donde no hay' preseneia de glaciares y se mantienen los valores en primavera y otono, además que la pluviosidad del área no presenta diferencias estacionales.

En $20 \mathrm{~m}$ el rango de variación estacional de temperatura mantiene una diferencia significativa. La salinidad tiende a aumentar con excepción de Inútil que mantiene su salinidad debido probablemente a procesos de mezcla producidos por el viento. La salinidad registrada en otoño es más baja que en primavera. El comportamiento hidrográfico de la capa de $20 \mathrm{~m}$ indicaría que este nivel esté influenciado pos los cambios estacionales.

El diagrama para el nivel de $50 \mathrm{~m}$ (Fig. 5) muestra una significativa disminución de la temperatura en otofio. La salinidad aumenta pero, el rango de ambos períodos estacionales disminuye. En el nivel de $100 \mathrm{~m}$ se aprecia un acercamiento de los va lores obtenidos en primavera y otoño. La relativa constancia de esta capa muestra la escasa influencia de los factores meteorológicos en ella.

\section{Discusion}

La distribución vertical de la temperatura presenta una termoclina superficial ubicada de $\operatorname{los} 40$ a los $80 \mathrm{~m}$ de profundidad.

La profundidad de la termoclina varía de acuerdo a las características de cada fiordo en particular. Sin embargo, hay una clara tendencia a aumentar en profundidad, a medida que los fiordos se acercan a la zona costera oceánica. Así,en Almirantazgo se ubica en $c a$. $40 \mathrm{~m}$ excepto en la boca donde no existe termoclina. Esta situación se presenta también en Inútil. En Otwiay la termoclina se 
ubica en los $50 \mathrm{~m}$ de profundidad y en Xaultegua alcanza los $70 \mathrm{~m}$. En general, la termoclina es coincidente con la haloclina. Bajo ella las características hidrográficas permiten dividir los fiordos estudiados en dos grupos: I) Aquelos que tienen una conexión directa con las áreas oceánicas y H) Los que se ubican en el interior del centinente, existiendo entre ellos y el oceáno una série de canales y áreas costeras (Pickard, 1967).

Los fiordos que tienen conexión directa con el ocáno, presentan una capa de mezcla de mayor espesos (i. e., $70 \mathrm{~m}$ ) y sus aguas profundas se identifican con valores de temperatura y salinidad con las aguas oceánico-costeras (Silva, 1977). Los valores más característicos de éstas se presentan en profundidades mayores de $100 \mathrm{~m}$ y correspenden a $8.4^{\circ} \mathrm{C}$ de temperatura y $c a .33 .00 \%$ o de salinidad.

Por otra parte, aquellos fiordos que no tienen conexión directa con el oceáno las salinidades y temperaturas de las aguas profundas disminuyen y presentan una significativa constancia con temperaturas de $c a .6 .0^{\circ} \mathrm{C}$ y salinidad de $c a .30 .60 \%$, valor que Brattström \& Dahl (1952) asignan a las aguas de superficie de las cuencas, para el área norte de los fiordos de Chile.

De acuerdo a la distribución de las aguas profundas, se puede caracterizar a G. Xaultegua como un seno con influencia de las aguas oceánico-costeras. En cambio, Seno Almirantazgo, Bahía Inútil y Seno Otway presentan una mayor dilución y por su dinámica durante el período otofial y primaveral se podría asumir que el intercambio con la zona costera es lento y las aguas profundas de estos senos mantendrían sus características a través del ciclo anual. Esta situación no implica la ausencia de mezcla vertical, probablemente los cambios estacionales de temperatura determinan importantes cambios en densidad de las capas superficiales de los fiordos estudiados y por tanto una mezcla activa, lo que explica- ría la presencia de un alto contenido de oxígeno en las aguas profundas y los valores relativamente bajos de salinidad para estos fiordos a pesar de la escasa pluviosidad de la zona.

La comparación de los resultados obtenidos en el período Primavera (HERO 72-4) y Otoño (HUDSON 70) reafirma la importancia de la temperatura en el comportamiento hidrográfico del área, tanto en el aspecto térmico, como en la distribución halina de los cuerpos de agua.

\section{Referências Bibliográficas}

BRATTSTROM, H. \& DAHL, E. 1951. Reports of the Lund University Chile Expedition 1948-49. 1. General account, lists of stations, hydrography. Lunds Univ. Arsskr., n. f. Avd. 2, 46 (8): 1-86.

CARRITT, D. E. \& CARPENTER, J. H. 1966. Comparison and evaluation of currently employed modification of the Winkler method for determining dissolved oxygen in seawater; a NASCO report. J. mar. Res., 24: 286-318.

CHUECAS, L. A. 1962. Investigaciones científicas. Ministerio de Agricultura y Pesca, Chile, $2: 1-11$.

PICKARD, G. L. 1967. Some oceanographic characteristics of the larger inlets of Southeast Alaska J. Fish. Res. Bd Can., 24 (7): $1475-1506$.

- - - 1971. Some physical oceanographic features of inlets of Chile. J. Fish. Res. Bd Can., 28 (8): 1077-1106. - 1973. Oceanography of the South Pacific 1972, comp. R. Fraser. Wellington, New Zealand National Comission for UNESCO.

SILVA, N. 1977. M.Śc. Thesis. Corvallis, Oregon State University.

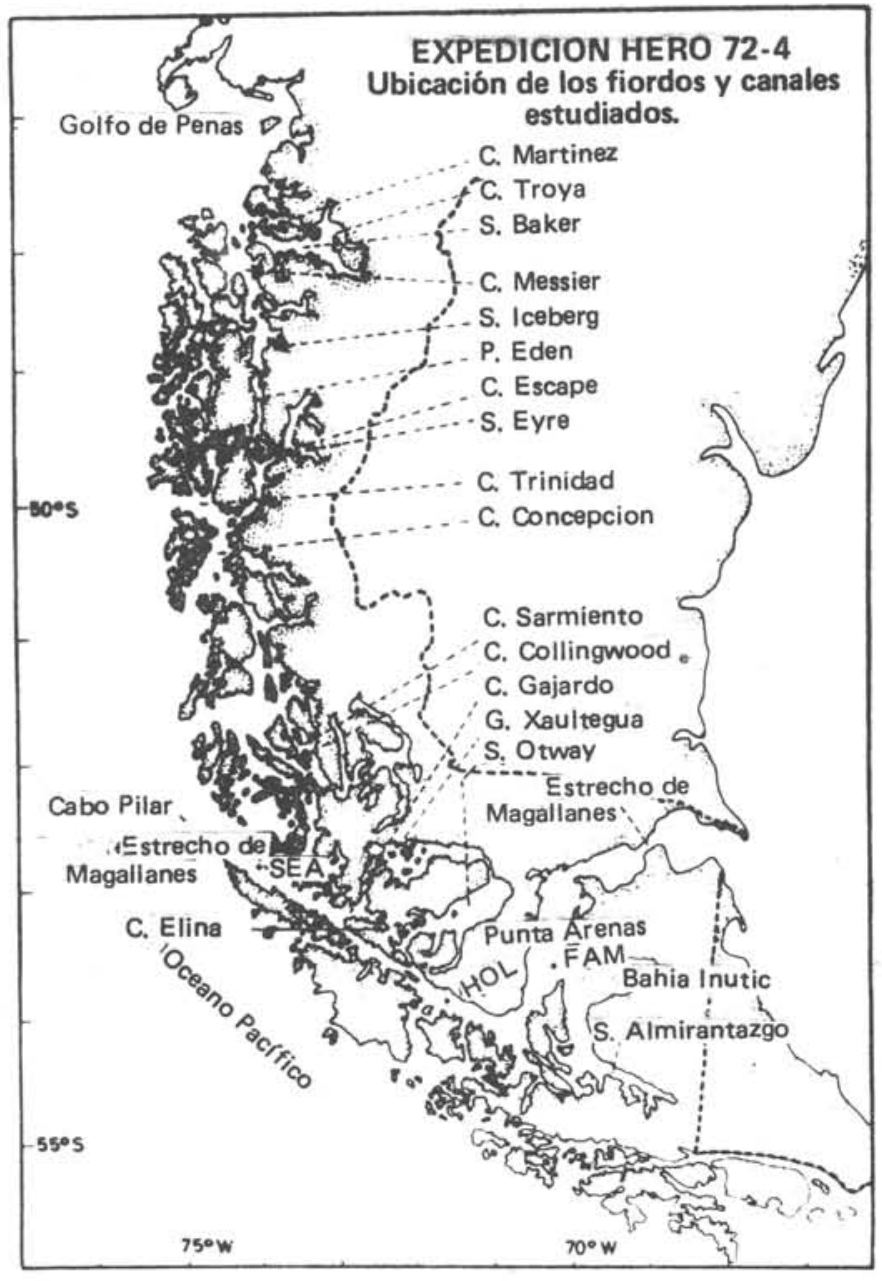

Fig, 1-El área de estudio comprendió los fiordos y canales ubicados entre Punta Arenas y Cabo Pilar. 

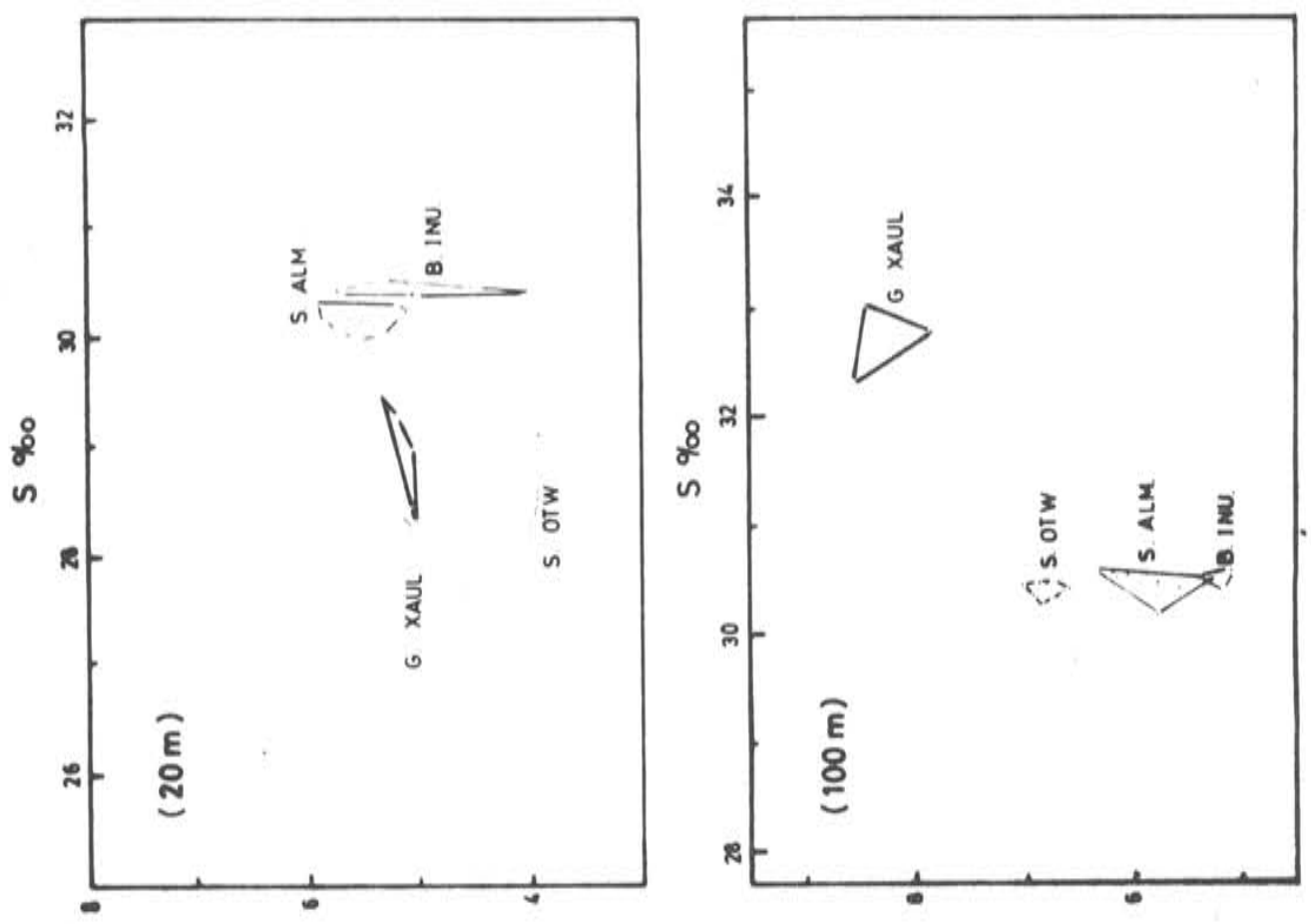

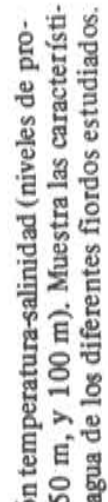
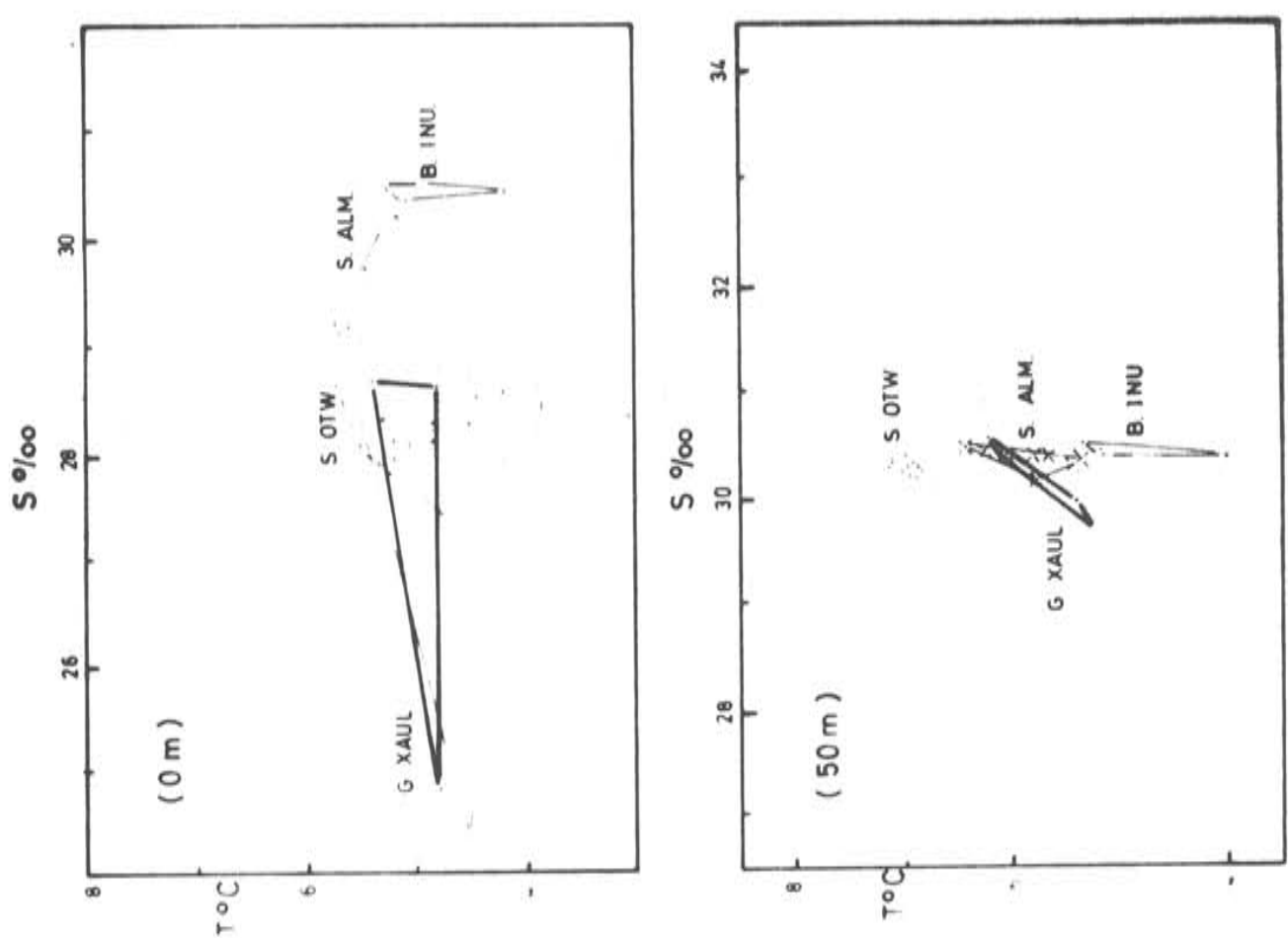

हैं

ํㅐㅇำ

Eี है

范

再范

尊急

ㄴ.

定 

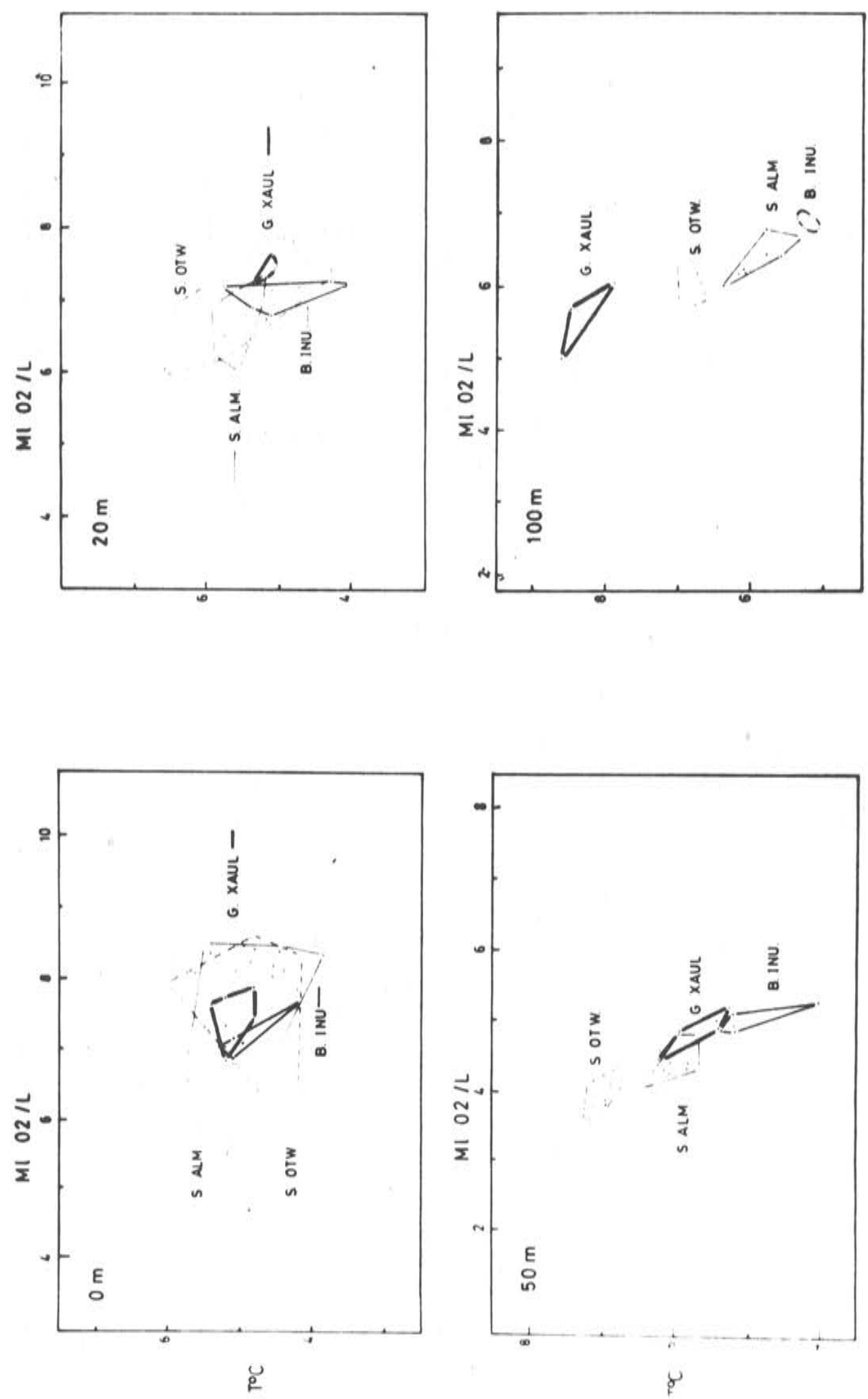

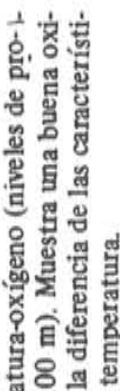

빙의 의 의

要

음 윰

递白禺

ำ

ชํㅇㅇ

ำ

to

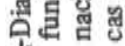

의

政

.



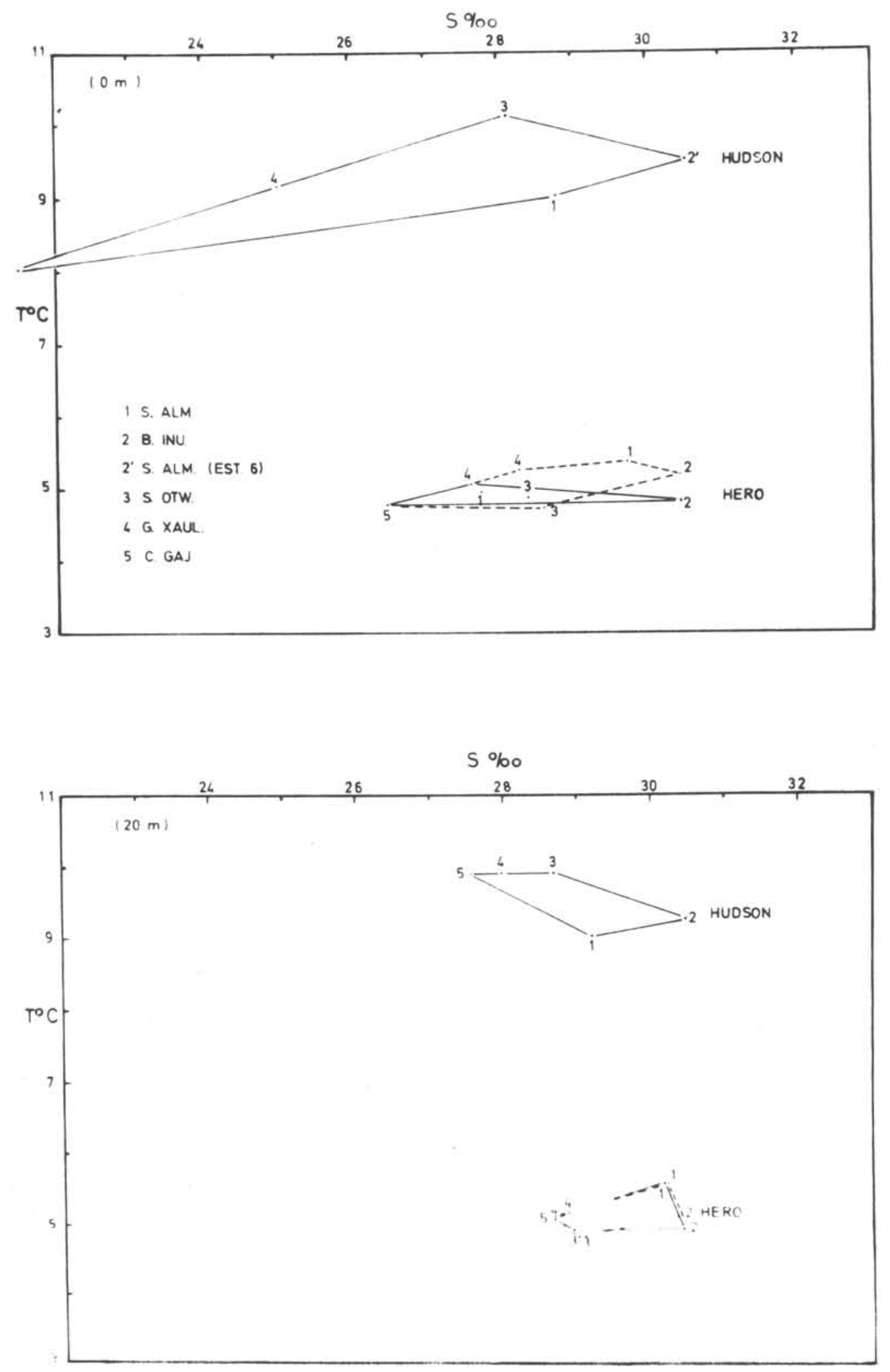

Fig. 4-Diagrama de correlación temperatura y salinidad. Muestra los promedios por seno para otofio (HUDSON 70) y primavera (HERO 72-4). Las líneas de trazos incluye en el promedio estaciones no contempladas en otofno y fueron consideradas de referencia. 

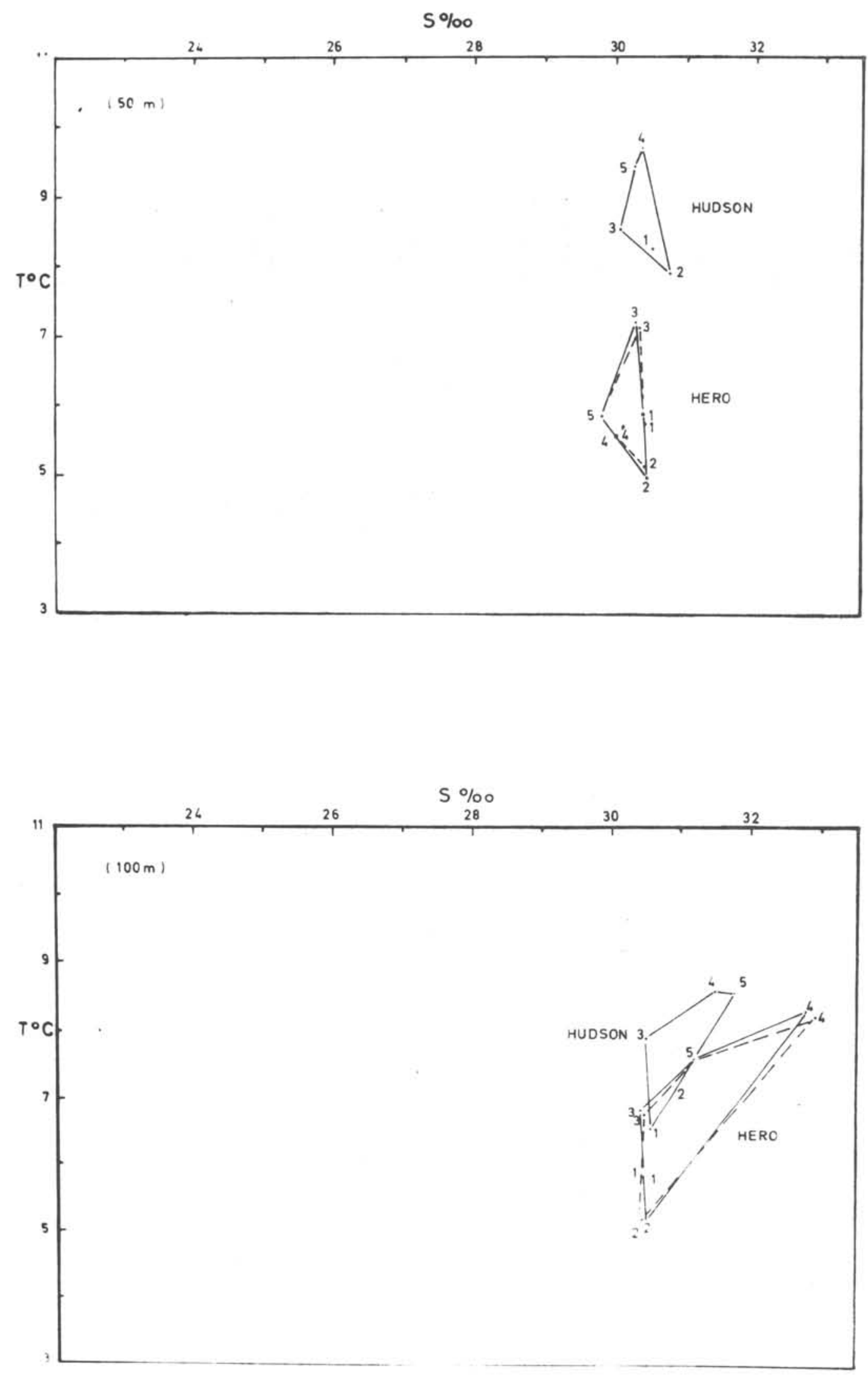

Fig. 5-Correlación temperatura, salinidad. Muestra los promedios por seno de otoño (HUDSON 70) y primavera (HERO 72-4). 